\title{
MAC Sub-Layer Design to Reduce Interference in LTE Networks
}

\author{
Weilian Su, Kim Hong Tan, Ric Romero and Tri Ha \\ Department of Electrical and Computer Engineering \\ Naval Postgraduate School \\ Monterey, CA 93940 \\ Telephone: (831) 656-3217 \\ Email: \{weilian, rnromero, ha\}@nps.edu
}

\begin{abstract}
In this paper, synergy between the physical layer and the Medium Access Control (MAC) layer of a Long Term Evolution (LTE) network is exploited to reduce the co-channel interference in both the forward and reverse channels. By doing such cross-layer optimization analysis, physical and MAC layer control decisions reach their full potential when they are designed in an integrated manner. The proposed solution focuses on the integration of the concepts of orthogonal frequency-division multiple access (OFDMA), sectorization, and Latin Square to improve the signal-to-interference ratio (SIR) with the most effective resource utilization. The solution seeks to achieve an optimum point of tradeoff between improvement in the SIR and the acceptable amount of the unutilized resources.
\end{abstract}

\section{INTRODUCTION}

From as early as the 19th century, through the vast contributions from the commercial, government, and military on technological developments and advancements, data communications have evolved from simple wired connections to modern robust wireless systems. The second decade of this century is seeing an increasing number of subscribers on wireless networks and a growing demand for higher data speed access and efficiency for web, streaming, and interactive video applications on the go. A Long Term Evolution (LTE) network is a fourth generation (4G) wireless network that is able to offer high-speed Internet access through the use of mobile phones. It is also able to handle large memory capacity that allows video phone calls and other multiple concurrent streaming systems to be accessed.

Future wireless data networks aim to offer high data rate coverage over large areas. With the orthogonal frequencydivision multiple access (OFDMA) technique adopted, interferences such as inter-symbol interference and intra-cell interference are minimized. This leaves co-channel interference (CCI) as the main problem that adversely affects the quality of service (QoS) of the data network.

Three main proposals to combat CCI as listed in 3GPP TR 25.814 [1] are CCI cancellation, CCI coordination, and CCI randomization. CCI cancellation only suppresses the dominating interference with the use of multiple antennas but requires extremely high complexity, while CCI randomization scrambles and interleaves the signal but does not reduce the interference. Finally, CCI coordination applies restrictions on both the downlink and uplink resource manager and on the transmitted power among the cells, making it the method with the most potential to reduce CCI. For instance, with an effective coordination and reuse of resources in a cellular system, the system capacity can be enhanced significantly. As such, with a lower rate at which the same frequency can be used again in the network, known as frequency reuse factor (FRF), more available bandwidth can be obtained for each cell. While this means that the FRF of 1 is desirable, most users would be seriously affected by strong CCI, especially users on the cell edge. One of the CCI mitigation schemes is to increase the cluster-order. This would lead to a decrease of the available bandwidth for each cell, resulting in restricted data transmissions and lower system spectrum efficiency among cells. Therefore, there is a need for careful resource planning and scheduling in the MAC sub-layer to reduce CCI optimally. Also, frequency reuse techniques described in [2]-[6], [10][12] reinforce such assertion.

In this paper, an investigation of the cross-layer optimization in an LTE network is performed to reduce the effect of CCI on both forward and reverse channels. With proper resource management in a coordinated scheduling strategy between cells, such as restrictions on the time/power allocation to the cell and adopting the frequency reuse scheme, it is hypothesized that co-channel interference can be mitigated.

The paper is organized as follows: Section II describes the proposed MAC sub-layer design and Section III evaluates the performance of the proposed design. Lastly, we conclude in Section IV.

\section{MAC Sub-LAYER DESIGN}

The scheduling strategy of the proposed MAC sub-layer design is described in Section II-A, and SIR analysis of the proposed scheduling strategy is provided in Section II-B.

\section{A. Scheduling Strategy}

To complement the sectoring technique used in LTE network, the proposed MAC sub-layer design aims to maximize interference diversity with the use of a Latin Square hopping scheme. Furthermore, as the Latin Squares are maintained by each Enhanced Node B ( $e N B$, which is also called enhanced 

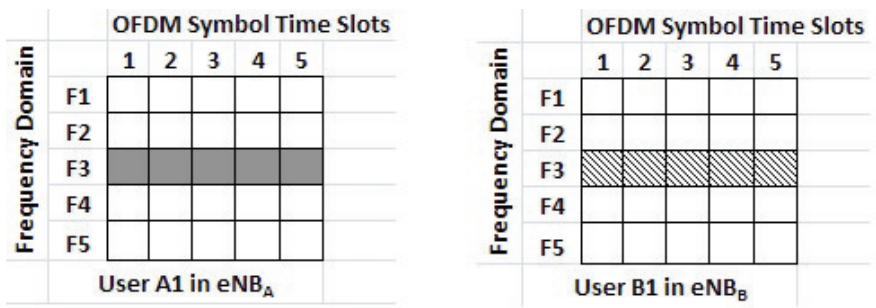

Fig. 1. An example of the worst case resource allocation for one user per $e N B$.
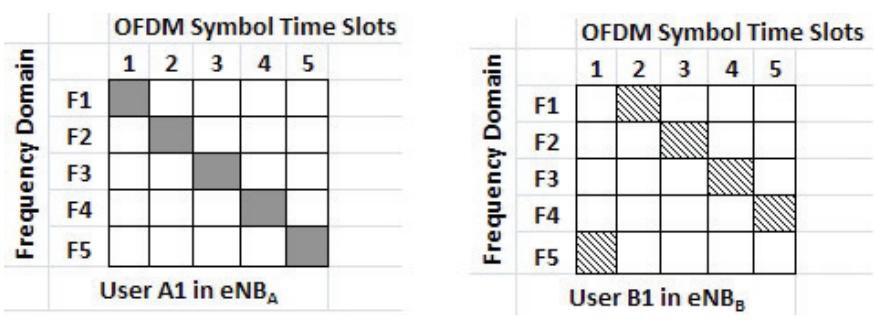

Fig. 2. An example of resource allocation for one user per cell with Latin Square.

basestation), where each virtual channel is able to hop over different sub-carriers at different OFDM symbol times, no single strong interference from a virtual channel can cause degradation in performance [7].

1) Brief Illustration: LTE [8] makes use of OFDMA to allocate the resource blocks to the users in both time and frequency domains such that different users can use the same sub-channel at different time while minimizing the chance of co-channel interference. However, in a worst case scenario, there may be two users, user $\mathrm{A} 1$ in $e N B_{A}$ and user B1 in $e N B_{B}$, being assigned the same resources at the same frequency and time, as shown in Figure 1. This is known as co-channel interference.

Therefore, the allocation of frequencies for the users should be as spread out as possible. Furthermore, these frequencies should be hopped every OFDM symbol time. In this manner, the hop patterns for the neighboring $e N B$ s are as far apart as possible, minimizing the chances of co-channel interference even more. This is the concept of the Latin Square frequency hopping scheme. An illustration of a possible resource allocation with Latin Square for the scenario in the previous paragraph is shown in Figure 2.

Each user sends its data using the allocated frequency channel in the particular time slot. As seen from the Latin Square for user A1 in $e N B_{A}$, colored in solid grey in Figure 2 , the assigned frequency channel changes each time frame to allow for frequency diversity. For example, the data sent out by user $\mathrm{A} 1$ in $e N B_{A}$ is first done in $\mathrm{F} 1$ in time slot 1 , then F2 in time slot 2, F3 in time slot 3, F4 in time slot 4, and F5 in time slot 5.

Similarly, for user B1 in $e N B_{B}$, shaded with stripes in Figure 2, the assigned frequency channel changes each time frame to allow for frequency diversity. The data sent out by user $\mathrm{B} 1$ in $e N B_{B}$ is first sent in $\mathrm{F} 5$ in time slot 1 , then $\mathrm{F} 1$ in time slot 2, F2 in time slot 3, F3 in time slot 4, and F4 in time slot 5 .

With this MAC sub-layer design of resource scheduling as illustrated in Figure 2, co-channel interference can be reduced significantly. The frequency hopping technique via Latin Square allows the $n$ sub-carriers allocated for the user to be as spread out as possible. Furthermore, these $n$ sub-carriers are hopped every OFDM symbol time. Although the amount of signal-to-interference ratio (SIR) improvement decreases as the number of users per Latin Square increases, due to the minimal chance of channel overlap, CCI is generally reduced.

As seen in $3 \mathrm{G}$ cellular networks [9] where a $60^{\circ}$-sectoring scheme is able to achieve better SIR performance than a $120^{\circ}$-sectoring scheme, the following analysis with a Latin Square frequency hopping scheme can only be done for the $60^{\circ}$-sectoring scheme. Furthermore, since the Cell Edge User (CEU) faces the worst SIR as compared to the Cell Center User (CCU), this analysis is performed on the CEU only for both forward and reverse channels.

To further illustrate the use of Latin Square scheme for forward channel analysis of CEU, the hopping pattern for the users in each $e N B$ is unique and is shown in Figure 3, where the star is the CEU. Mapping the matrices together as shown in Figure 4, we see that there is only one instance when the users use the same frequency and time slot. This clearly shows that with Latin Square Scheme used, the probability of overlapped channels is reduced, reducing the effect of CCI.

2) Virtual Channel Assignment Approach (Scenario 1): Assuming a channel bandwidth of $3 \mathrm{MHz}$ with 15 resource blocks (a resource block has 12 subcarriers) and for a Latin Square of size 5, we see that a family of four distinct quasiorthogonal Latin Squares is available: one for the $e N B$ under consideration and three for three co-channel $e N B \mathrm{~s}$. According to [7], we can create these Latin Squares when the square size is prime, allowing each $e N B$ a unique Latin square.

The channel bandwidth of $3 \mathrm{MHz}$ with 15 resource blocks (15 12-subcarrier-blocks over 20 time slots per frame) can accommodate a total of 12 identical Latin Squares. With one virtual channel assigned to each user, 12 Latin squares can support a maximum of 60 users per sector per $e N B$. In the case of the maximum number of users, the probability of co-channel interference is the highest, and the utilization of the virtual channel is also at the highest. This illustration is depicted in Figure 5.

Assuming the same parameters where the channel bandwidth is $3 \mathrm{MHz}$ with 15 resource blocks, but this time the Latin Square size is 7, we see that a family of six distinct quasi-orthogonal Latin Squares is available: one for the $e N B$ under consideration, three for three co-channel $e N B \mathrm{~s}$, and two unused. Due to the frame length of 20 time slots and the frequency constrained by the number of resource blocks defined in each channel bandwidth, each frame can accommodate two full Latin Squares and five partial Latin Squares, where some of the resource blocks in a Latin Square are not used. These unusable resource blocks are shaded in Figure 6. The two 


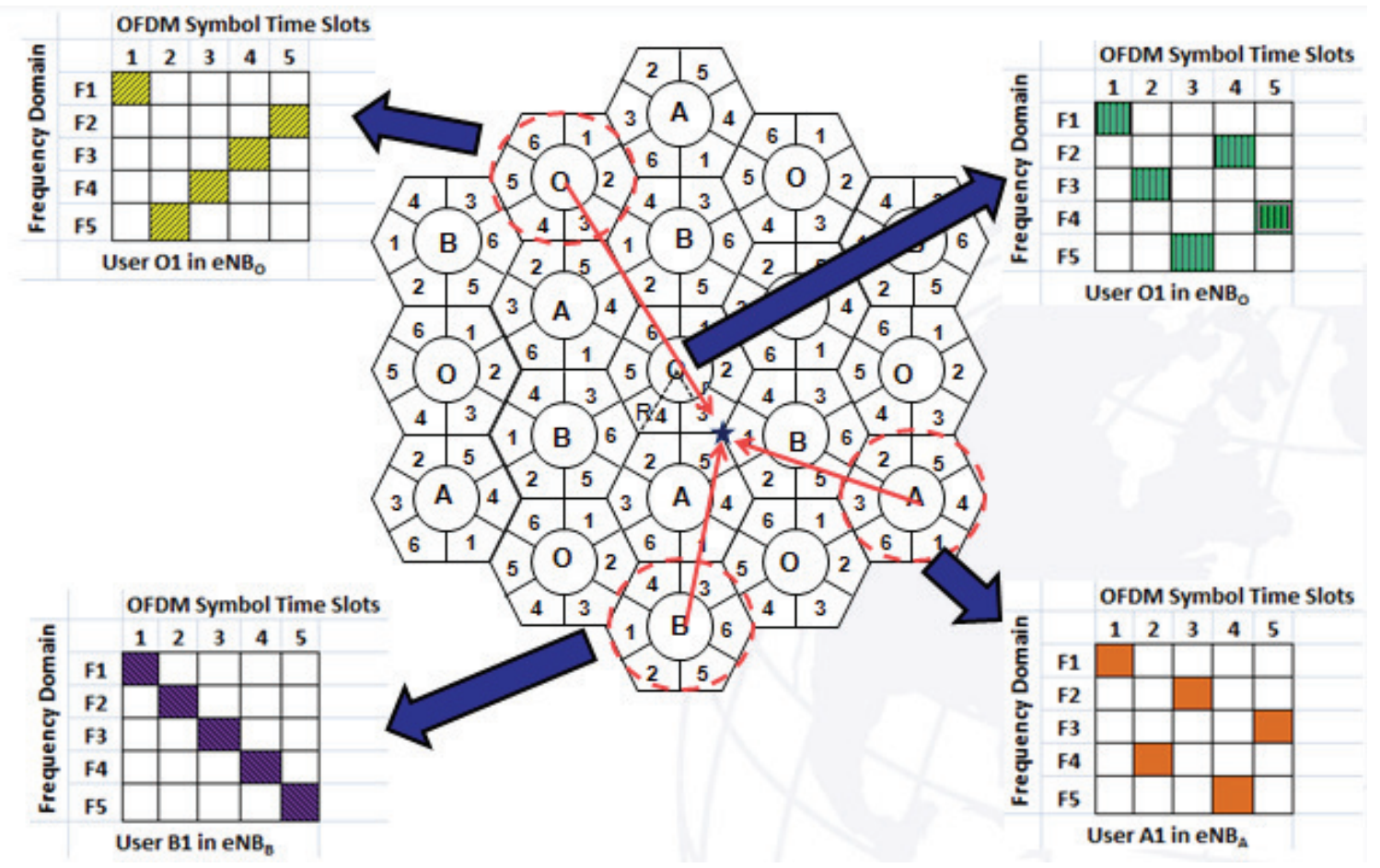

Fig. 3. Unique hopping patterns for different $e N B$ s with Latin Square scheme.

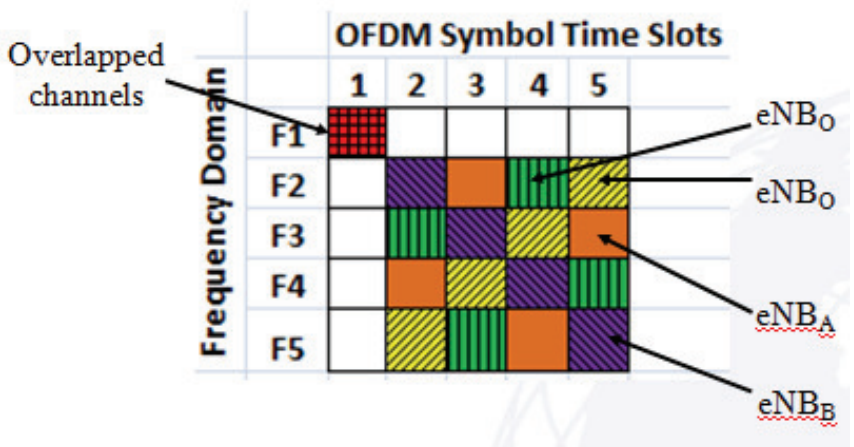

Fig. 4. Mapped matrices.

partial Latin Squares on the right border can still serve seven users each, but each user is only allocated six timeslots with seven 12-subcarrier blocks instead of seven timeslots as in the full Latin Square. Similarly, two partial Latin Squares on the bottom border can also serve seven users each, but each user is only allocated one timeslot and only one 12-subcarrier block. Lastly, the partial Latin Square on the bottom right border can only serve six users with one timeslot each and only one 12-subcarrier block. In this case, a maximum of 62 users per sector per $e N B$ can be supported, but only 28 users receive seven resource blocks each, 14 users with six resource blocks each, and 20 users with one resource block each.

In the case of the maximum number of users, the probability of co-channel interference is the highest, and the utilization of the virtual channels is also at the highest.
Time slots per frame

\begin{tabular}{|c|c|c|c|c|c|c|c|c|c|c|c|c|c|c|c|c|c|c|c|c|}
\hline & 1 & 2 & 3 & 4 & 5 & 6 & 7 & 8 & 9 & 10 & 11 & & & & & & 17 & & & \\
\hline & 0 & 1 & 2 & 3 & 4 & 0 & 1 & 2 & 3 & 4 & 0 & 1 & 2 & 3 & 4 & 0 & 1 & 2 & 3 & 4 \\
\hline & 2 & 3 & 2 & 0 & 1 & 2 & 3 & 4 & 0 & 1 & 2 & 3 & 4 & 0 & 1 & 2 & 3 & 4 & 0 & 1 \\
\hline & 4 & 0 & 1 & 2 & 3 & 4 & 0 & 1 & 2 & 3 & 4 & 0 & 1 & 2 & 3 & 4 & 0 & 1 & 2 & 3 \\
\hline & 1 & 2 & 3 & 4 & 0 & 1 & 2 & 3 & 4 & 0 & 1 & 2 & 3 & 4 & 0 & 1 & 2 & 3 & 4 & 0 \\
\hline & 3 & 4 & 0 & 1 & 2 & 3 & 4 & 0 & 1 & 2 & 3 & 4 & 0 & 1 & 2 & 3 & 4 & 0 & 1 & 2 \\
\hline & 0 & 1 & 2 & 3 & 4 & 0 & 1 & 2 & 3 & 4 & 0 & 1 & 2 & 3 & 4 & 0 & 1 & 2 & 3 & 4 \\
\hline & 2 & 3 & 4 & 0 & 1 & 2 & 3 & 4 & 0 & 1 & 2 & 3 & 4 & 0 & 1 & 2 & 3 & 4 & 0 & 1 \\
\hline & 4 & 0 & 1 & 2 & 3 & 4 & 0 & 1 & 2 & 3 & 4 & 0 & 1 & 2 & 3 & 4 & 0 & 1 & 2 & 3 \\
\hline & 1 & 2 & 3 & 4 & 0 & 1 & 2 & 3 & 4 & 0 & 1 & 2 & 3 & 4 & 0 & 1 & 2 & 3 & 4 & 0 \\
\hline & 3 & 4 & 0 & 1 & 2 & 3 & 4 & 0 & 1 & 2 & 3 & 4 & 0 & 1 & 2 & 3 & 4 & 0 & 1 & 2 \\
\hline & 0 & 1 & 2 & 3 & 4 & 0 & 1 & 2 & 3 & 4 & 0 & 1 & 2 & 3 & 4 & 0 & 1 & 2 & 3 & 4 \\
\hline & 2 & 3 & 4 & 0 & 1 & 2 & 3 & 4 & 0 & 1 & 2 & 3 & 4 & 0 & 1 & 2 & 3 & 4 & 0 & 1 \\
\hline & 4 & 0 & 1 & 2 & 3 & 4 & 0 & 1 & 2 & 3 & 4 & 0 & 1 & 2 & 3 & 4 & 0 & 1 & 2 & 3 \\
\hline & 1 & 2 & 3 & 4 & 0 & 1 & 2 & 3 & 4 & 0 & 1 & 2 & 3 & 4 & 0 & 1 & 2 & 3 & 4 & 0 \\
\hline & 3 & 4 & 0 & 1 & 2 & 3 & 4 & 0 & 1 & 2 & 3 & 4 & 0 & 1 & 2 & 3 & 4 & 0 & 1 & 2 \\
\hline
\end{tabular}

Fig. 5. An example of resource allocation for a channel bandwidth of $3 \mathrm{MHz}$ per sector per $e N B$, with Latin Square size 5 (Scenario 1).

3) Resource Block Assignment Approach (Scenario 2): Again, assuming a channel bandwidth of $3 \mathrm{MHz}$ with 15 resource blocks and the users assigned one resource block per timeslot each, we see that the maximum number of users supported is 300 , i.e., $20 \times 15$. In order to minimally support this number of users, a Latin Square of size 19, which is the next prime of square root of 300 , is used. At this size, however, the probability of co-channel interference is high. 
Time slots per frame

\begin{tabular}{lllllllllllllll|l|l|l|l|l|l|l|l}
1 & 2 & 3 & 4 & 5 & 6 & 7 & 8 & 9 & 10 & 11 & 12 & 13 & 14 & 15 & 16 & 17 & 18 & 19 & 20
\end{tabular}

\begin{tabular}{l|l|l|l|l|l|l|l|l|l|l|l|l|l|l|l|l|l|l|l|}
0 & 1 & 2 & 3 & 4 & 5 & 6 & 0 & 1 & 2 & 3 & 4 & 5 & 6 & 0 & 1 & 2 & 3 & 4 & 5 \\
\hline
\end{tabular}

\begin{tabular}{llllllllllllllllllllllll}
2 & 3 & 4 & 5 & 6 & 0 & 1 & 2 & 3 & 4 & 5 & 6 & 0 & 1 & 2 & 3 & 4 & 5 & 6 & 0 \\
\hline & 5 & 6 & 0 & 1 & 2 & 3 & 4 & 5 & 6 & 0 & 1 & 2 & 3 & 4 & 5 & 6 & 0 & 1 & 2
\end{tabular}

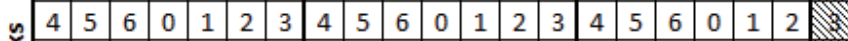

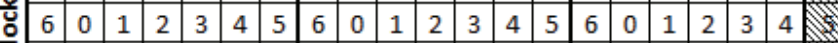

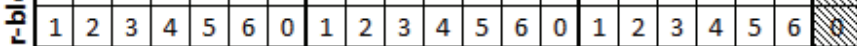

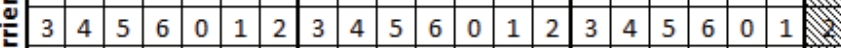

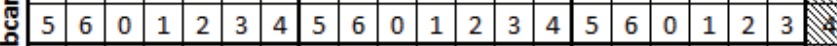

寻 0

ํ่

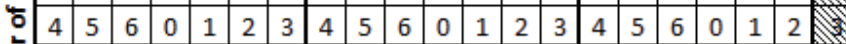

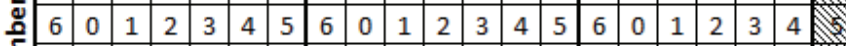

E

\begin{tabular}{|l|l|l|l|l|l|l|l|l|l|l|l|l|l|l|l|l|l|l|l|}
\hline 3 & 4 & 5 & 6 & 0 & 1 & 2 & 3 & 4 & 5 & 6 & 0 & 1 & 2 & 3 & 4 & 5 & 6 & 0 & 1
\end{tabular}

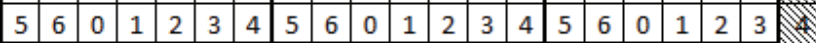

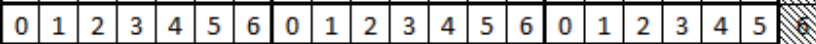

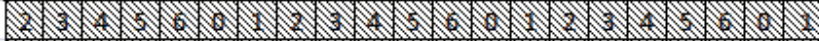

4 M M H W W W W W W

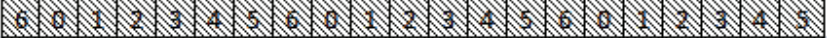

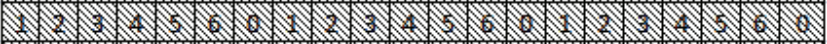

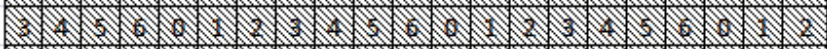

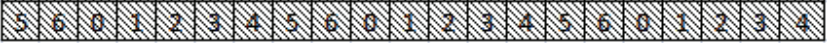

Fig. 6. An example of resource allocation for a channel bandwidth of $3 \mathrm{MHz}$ per sector per $e N B$, with Latin Square size 7 (Scenario 1).

This is because the probability of multiple users being assigned the same resource block increases when the number of users in the network increases. As such, the best case with the lowest effect of co-channel interference is the next prime number of the square root of the number of users multiplied by the number of interfering sources plus one, which indicates the reference source.

For example, if the number of interfering sources is three, then to have the lowest effect of co-channel interference, the size of the Latin Square in this case is the next prime number of the square root of $(300 \times 4)$, which equates to 37. The size of the Latin Square is then varied from the prime numbers between 19 and 37, which are 19, 23, 29, 31, and 37 , to investigate the behavior of co-channel interference and resource utilization. Although a bigger Latin Square size means a lower effect of co-channel interference, it also means that the utilization of the resources decreases. Taking Latin Squares of sizes 23 and 29 as shown in Figure 7, we see that each lighter shaded small square represents one resource block per user (300 of them in this case and bounded by the frame length of 20 time slots and channel frequency). The darker shaded small squares are the unutilized resource blocks from the remaining Latin Square sizes of 23 and 29, respectively.

\section{B. SIR Analysis of Proposed Scheduling Strategy for $60^{\circ}$ sectoring}

The SIR equation derived for $60^{\circ}$-sectoring (not included in this paper due to space) has the general form

$$
S I R=\frac{P_{\text {desired }}}{\left(p_{1} I_{1}\right)+\left(p_{2} I_{2}\right)+\left(p_{3} I_{3}\right)+\ldots},
$$

where $P_{\text {desired }}$ is desired received power, $p_{i}$ ( $i$ starts from 1 to infinity) is the probability of the interference source, and $I_{i}$ is the power of the interference source $i$.

Without the use of the Latin Square frequency hopping scheme to diversify the sub-carriers assignment, the worst case where an $e N B$ becomes an interfering source has a probability $p_{i}$ of 1 . However, with the use of the Latin Square frequency hopping scheme, the resources are allocated to the users in a staggered manner as shown previously in Figure 4. This means that the probability $p_{i}$ of two users in two $e N B \mathrm{~s}$ (one user per $e N B$ ) being allocated the same resources in the same frequency and time symbol is reduced. Certainly, this probability $p_{i}$ of the $e N B$ becoming an interfering source increases when the number of users per Latin Square increases or when the size of Latin Square decreases.

As shown in Figure 8, there are three interfering sources in the second tier, from cells $\mathrm{A}, \mathrm{B}$, and $\mathrm{O}$ (cell on the upper left of the figure). The SIR for the CEU in the forward channel for the $60^{\circ}$-sectoring becomes

$$
\begin{aligned}
& S I R_{C E U, 60^{\circ}}= \\
& \frac{P_{T, O}}{p_{O}(4)^{-n} P_{T, O}^{\prime}+p_{A}(\sqrt{7})^{-n} P_{T, A}+p_{B}(\sqrt{7})^{-n} P_{T, B}}
\end{aligned}
$$

where $P_{T, O}$ is the transmitted power of $e N B_{O}, P_{T, O}^{\prime}$ is the transmitted power of $e N B_{O}$ on the top left, $P_{T, A}$ is the transmitted power of $e N B_{A}, P_{T, B}$ is the transmitted power of $e N B_{B}, n$ is the path loss component factor and $p_{O}, p_{A}$, and $p_{B}$ are the probabilities of interferences from cells $\mathrm{A}, \mathrm{B}$, and $\mathrm{O}$ from the top left.

Similarly, for the reverse channel for $60^{\circ}$-sectoring, there are also three interfering sources in the second tier as shown in Figure 9. The desired user equipment is located at the corner of the outer cell in cell $\mathrm{O}$ (shown as the star). Since cell edge users adopt a sectoring approach, only cells A and B at the bottom right in the first tier which are facing the intended sector (sub-channel 3) contribute to the CCI. However, since the sectors using sub-channel 3 are facing away from the intended sector, the analysis is moved on to the second tier, where the bottom cells A, B, and $\mathrm{O}$ have their sector with subchannel 3 facing the intended sector. The positions where the user equipment interfere most are marked as squares. The SIR for the cell edge in the reverse channel for the $60^{\circ}$-sectoring becomes

$$
\begin{aligned}
& S I R_{C E, 60^{\circ}}= \\
& \frac{P_{T, O}}{p_{O}\left(3+\frac{r}{R}\right)^{-n} P_{T, O}^{\prime}+p_{A}\left(\frac{3 \sqrt{3}}{2}\right)^{-n} P_{T, A}+p_{B}\left(\frac{3 \sqrt{3}}{2}\right)^{-n} P_{T, B}}
\end{aligned}
$$

where $C E$ stands for cell edge area, and $r$ and $R$ are the inner and outer cell radii of a cell, respectively. 

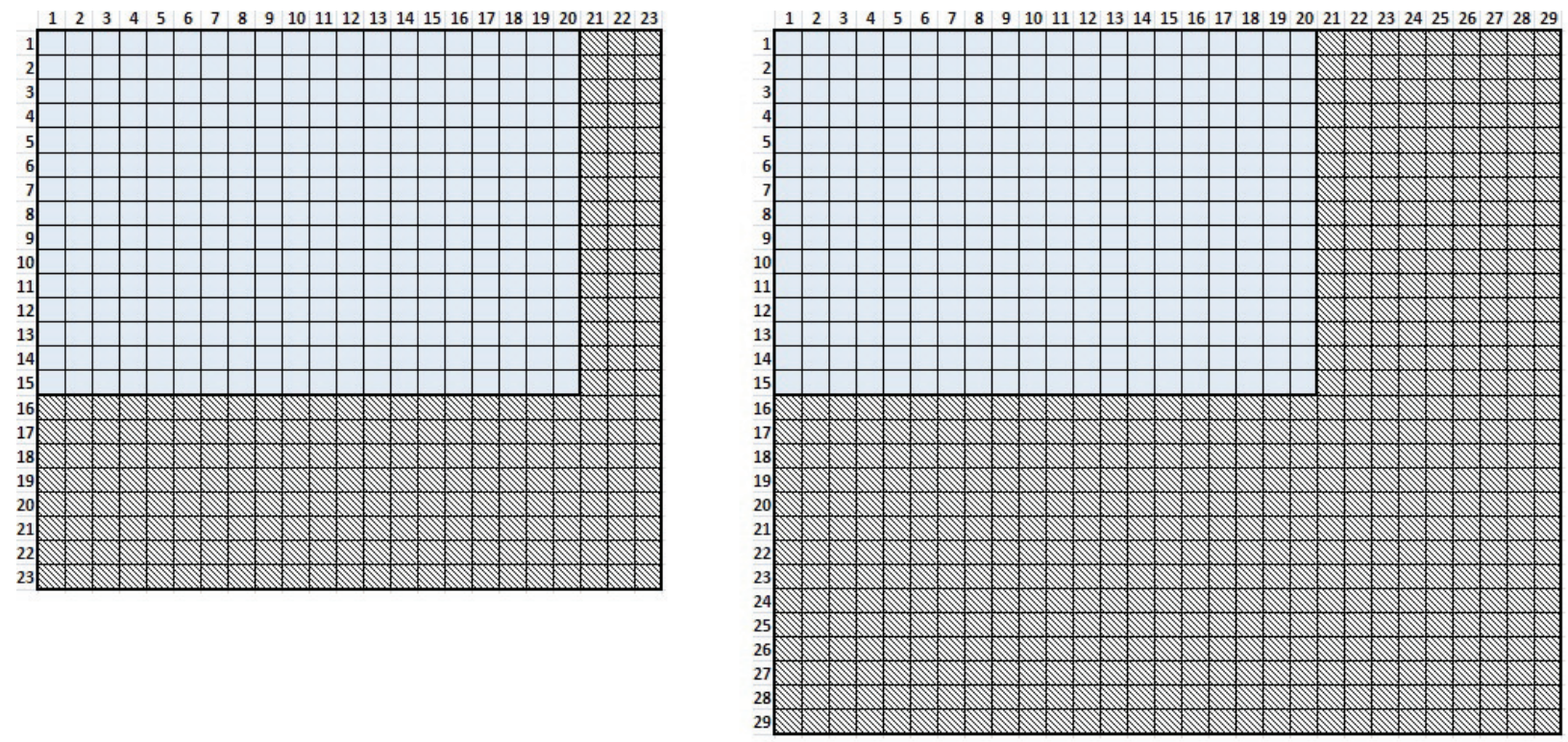

Fig. 7. An example of resource allocation for a channel bandwidth of $3 \mathrm{MHz}$ per sector per $e N B$, with various Latin Square sizes (Scenario 2).

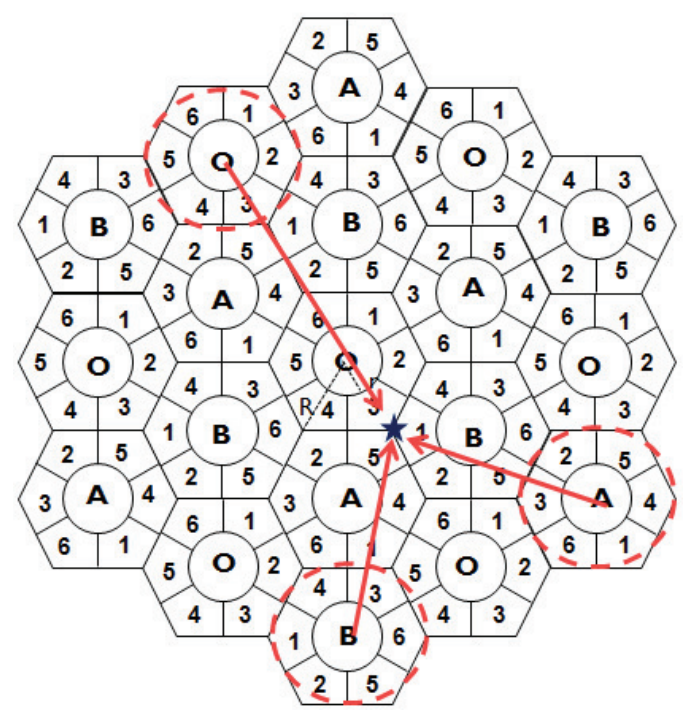

Fig. 8. Forward channel interference experienced by CEU.

\section{PERFormance EVAluation}

\section{A. Test Setup and Objective}

It is observed from the SIR equations, Equations (2) and (3), that the SIR is dependent upon the path loss exponent, whose value is normally in the range of two to four, where two is for propagation in free space and four is for relatively lossy environments. For the purpose of the simulations for this paper, a path loss exponent of four is used. A simulation code in Matlab was written to calculate the average SIR.

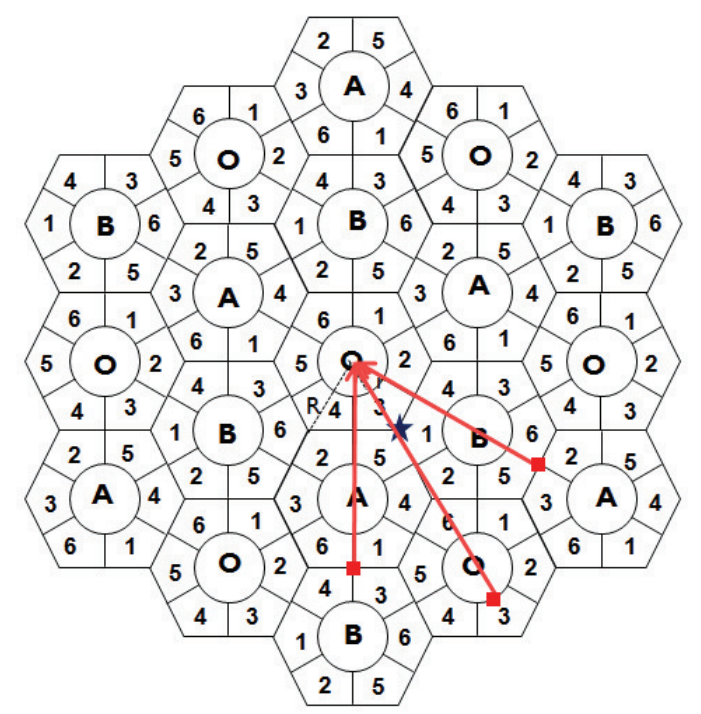

Fig. 9. Reverse channel interference analysis for edge area.

\section{B. Virtual Channel Assignment Approach (Scenario 1) for the Forward Channel}

Using Equation (2), we obtain the behavior of the SIR and the resource allocation for the channel bandwidths of $10 \mathrm{MHz}$ for the forward channel shown in Figures 10 and 11. The number of users per Latin Square is varied between 5 and 7 to illustrate the SIR and the resource utilization behavior.

From Figures 10 and 11, it can be observed that with the Latin Square scheme applied, the SIR improves in general. In fact, by looking at the number of users from one to five only for both Latin Square sizes of 5 and 7, the SIR improves more with the Latin Square size 7. This shows that with a bigger Latin Square size, the probability that users are assigned 


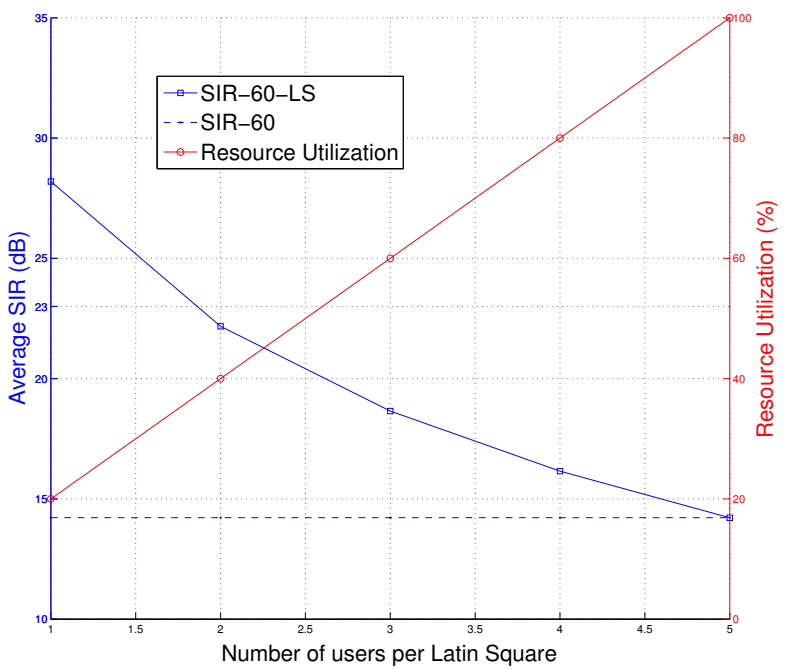

Fig. 10. SIR performance and resource utilization for forward channel, with $10 \mathrm{MHz}$ channel bandwidth per sector, Latin Square (LS) size 5.

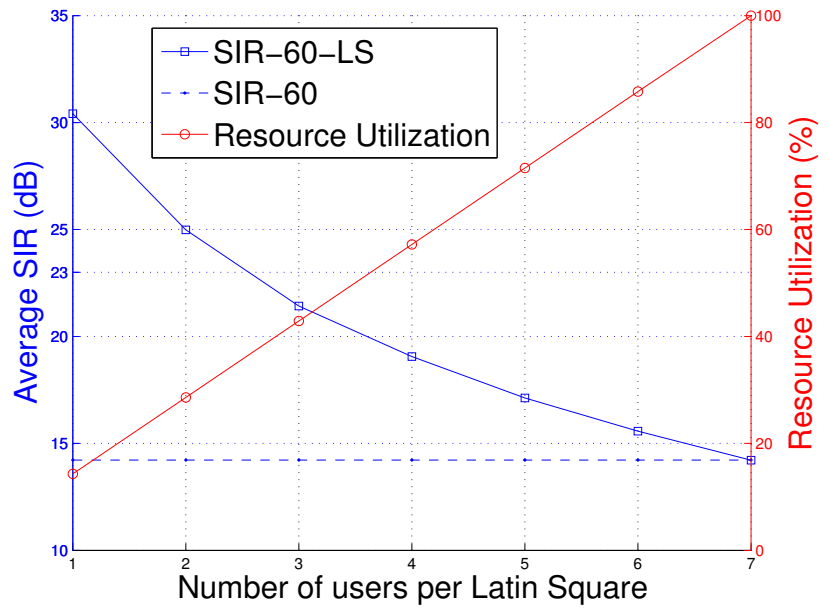

Fig. 11. SIR performance and resource utilization for forward channel, with $10 \mathrm{MHz}$ channel bandwidth per sector, Latin Square size 7.

the same virtual channel is reduced as the number of virtual channels assigned is increased from five to seven. Having said this, we note that with a range of users from one to five, the resource utilization for Latin Square size 7 drops by about $20 \%$ of that of Latin Square size 5. Also, the number of users supported is reduced since a transmission frame of 20 time slots and 15 resource blocks fits a smaller number of $(7 \times 7)$ Latin Squares as compared to $(5 \times 5)$.

The extent that the SIR improves is greater as the number of users per Latin Square decreases. This makes sense because, when there are fewer users per $e N B$, the probability of the users being assigned the same virtual channel decreases, reducing the effect of co-channel interference. On the other hand, when the number of users per Latin Square increases,

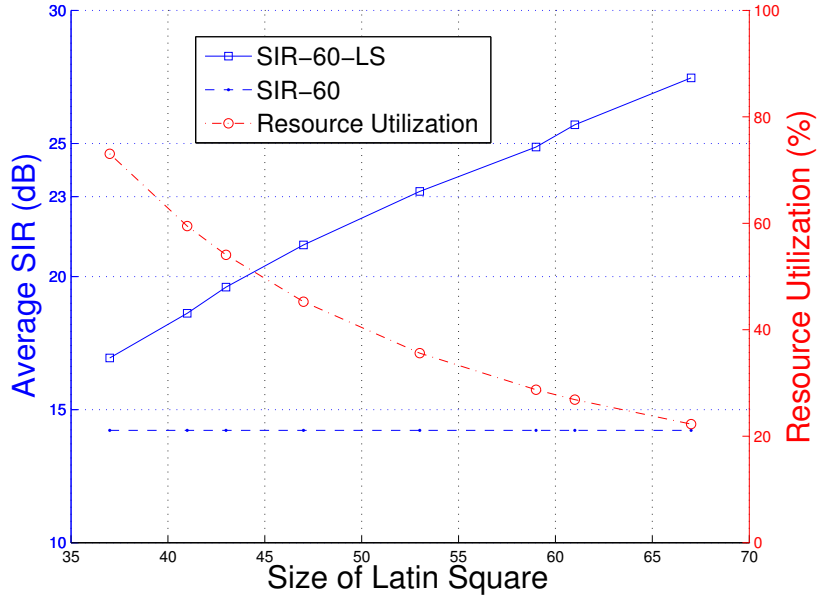

Fig. 12. SIR performance and resource utilization for forward channel, with $10 \mathrm{MHz}$ channel bandwidth per sector.

the resources are better utilized, therefore, the increase in the resource utilization.

\section{Resource Block Assignment Approach (Scenario 2) for the Forward Channel}

Using Equation (2), we show the behavior of the SIR and the resource allocation for $10 \mathrm{MHz}$ channel bandwidths for the forward channel in Figure 12.

It can be observed that with the Latin Square scheme applied, the SIR improves in general. In fact, the SIR improves more as the size of the Latin Square increases. This makes sense because when the Latin Square is large, the probability that the users are assigned the same resources decreases, reducing the effect of co-channel interference. On the other hand, when the size of the Latin Square increases, the resources are less utilized, therefore, there is a decrease in the resource utilization.

\section{Virtual Channel Assignment Approach (Scenario 1) for the Reverse Channel}

The same analysis is applied in the reverse channel, and by using Equation (3), the behavior of the SIR and the resource allocation for $10 \mathrm{MHz}$ channel bandwidth for the reverse channel are shown in Figures 13 and 14. Again, the number of users per Latin Square is varied between 5 and 7 to illustrate the SIR and the resource utilization behavior.

From Figures 13 and 14, the same observation on the forward channel can be made for the reverse channel. When the Latin Square scheme is applied, the SIR improves in general. By comparing the SIR for the one to five users range for Latin Square sizes 5 and 7, we see that the SIR improves more with Latin Square of size 7. This shows that with a larger Latin Square size, the probability that the users are assigned the same virtual channel is reduced as the number of virtual channels assignment is increased from five to seven. It is also important to note that from the user range of one to five, the 


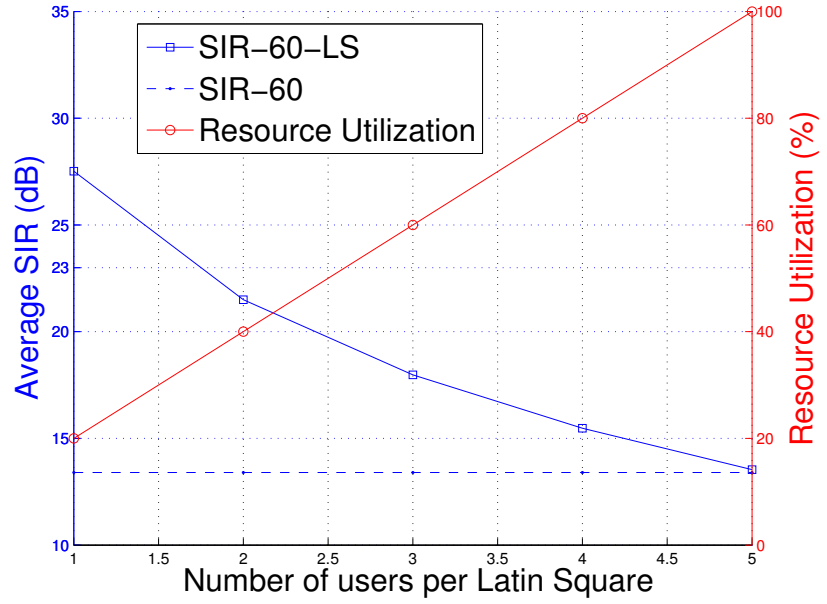

Fig. 13. SIR performance and resource utilization for reverse channel, with $10 \mathrm{MHz}$ channel bandwidth per sector, Latin Square size 5.

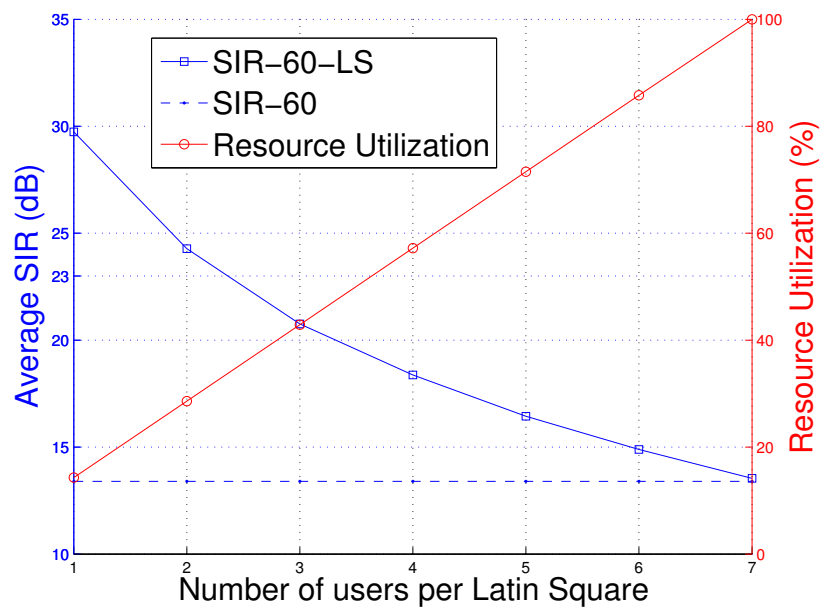

Fig. 14. SIR performance and resource utilization for reverse channel, with $10 \mathrm{MHz}$ channel bandwidth per sector, Latin Square size 7.

resource utilization for Latin Square size 7 drops by about $20 \%$ of that of Latin Square size 5.

Furthermore, the extent that the SIR improves is greater as the number of users per Latin Square decreases. When there are fewer users per $e N B$, the probability that the users are assigned the same virtual channel decreases, reducing the effect of co-channel interference. On the other hand, when the number of users per Latin Square increases, the resources are better utilized, therefore, the increase in the resource utilization.

\section{E. Resource Block Assignment Approach (Scenario 2) for the Reverse Channel}

By using Equation (3), we show the behavior of the SIR and the resource allocation for $10 \mathrm{MHz}$ channel bandwidth for the reverse channel in Figure 15.

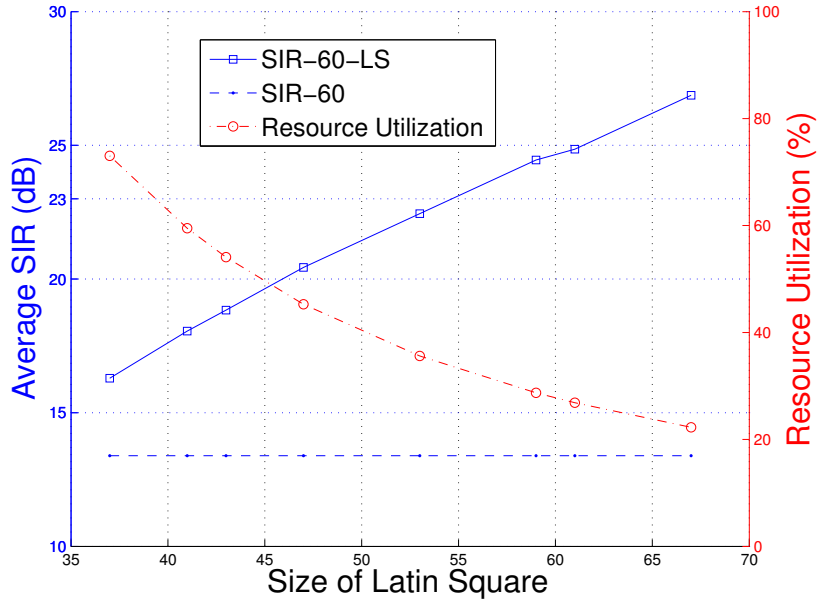

Fig. 15. SIR performance and resource utilization for reverse channel, with $10 \mathrm{MHz}$ channel bandwidth per sector.

In Figure 15, it can be observed that with the Latin Square scheme applied, the SIR again improves in general. In fact, the SIR improves more as the size of the Latin Square increases. This makes sense because when the Latin Square is larger, the probability that the users are assigned the same resources decreases, reducing the effect of co-channel interference. On the other hand, when the size of the Latin Square increases, the resources are less utilized, therefore, the decrease in the resource utilization.

\section{CONCLUSION}

The paper proposed a MAC sub-layer design to reduce the co-channel interference. It utilizes the Latin Squares to provide resource allocations. From simulations, the proposed scheduling strategy is effective for an LTE network and is able to improve SIR when the size of the Latin Square increases. More simulations need to be done to evaluate the performance under different transmission bandwidth, e.g., $3 \mathrm{MHz}$ and 20 MHz.

\section{REFERENCES}

[1] Physical layer aspect for evolved Universal Terrestrial Radio Access (UTRA), 3GPP TR 25.814, 2006.

[2] W. Fan et al., "An Inter-Cell Interference Coordination Technique Based on Users' Ratio and Multi-Level Frequency Allocations," Proc. of International Conference on Wireless Communications, Networking and Mobile Computing, pp.799-802, Sept. 2007.

[3] Z. Xie and B. Walke, "Frequency Reuse Techniques for Attaining Both Coverage and High Spectral Efficiency in OFDMA Cellular Systems," Proc. of Wireless Communications and Networking Conference, pp.1-6, April 2010.

[4] M. Porjazoski and B. Popovski, "Analysis of Intercell interference coordination by Fractional frequency reuse in LTE," Proc. of International Conference on Software, Telecommunications and Computer Networks (SoftCOM), pp.160-164, Sept. 2010.

[5] M. Porjazoski and B. Popovski, "Contribution to analysis of Intercell interference coordination in LTE: A fractional frequency reuse case," Proc. of Mobile Congress, pp.1-4, Oct. 2010. 
[6] Y. Yu et al., "Performance analysis of soft frequency reuse for intercell interference coordination in LTE networks," Proc. of International Symposium on Communications and Information Technologies (ISCIT), pp.504-509, Oct. 2010

[7] D. Tse and P. Viswanath, Fundamentals of Wireless Communication, Cambridge University Press, 2005.

[8] LTE, Evolved Universal Terrestrial Radio Access (E-UTRA), User Equipment (UE) radio transmission and reception, 3GPP TS 36.101 version 10.6.0 Release 10, 2011.

[9] T. T. Ha, Theory and Design of Digital Communication Systems, Cambridge University Press, 2011.

[10] S. Iwelski, et. al., "Analysis of interference-aware receivers in heterogeneous LTE networks," IEEE/CIC International Conference on Communications in China, XI'AN, China, August 2013.

[11] D. Lopez-Porez and H. Claussen, "Improved frequency reuse through sector offset configuration in LTE Heterogeneous Networks," IEEE International Conference on Communications, Sydney, Australia, June 2014.

[12] C. Sun, et. al., "Adaptive partial frequency reuse in LTE-Advanced relay networks," IEEE International Performance Computing and Communications Conference, Nanjing, China, December 2015. 